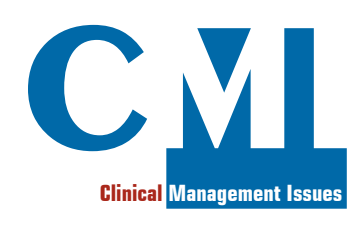

\title{
Telemedicine: Current Status and Future Perspectives
}

Keywords: Telemedicine; Telehealth; Healthcare

CMI 2021; 15(1): 41-43

http://dx.doi.org/10.7175/cmi.v15i1.1511
Telemedicine and telehealth are often considered synonyms, but while telemedicine refers particularly to clinical patient care, telehealth includes all the educational, administrative, and other non-clinical healthcare activities.

Although the roots of telemedicine date quite far back, the modern era of telemedicine started in 1968: in that date, the Massachusetts General Hospital (MGH) became the first hospital-based multispecialty telemedicine practice offering remote clinical examinations to travelers and airport workers at Logan International Airport [1].

Historically, telemedicine has been applied in several medical specialties such as psychiatry, cardiology, pediatrics, gynecology, geriatrics, with general patient satisfaction [2-9].

With the evolving technology, more specific applications of telemedicine have been developed, e.g., tele-dermatoscopes (pecific general patient cameras mounted on webcams or smartphones and controlled by app) have been used to monitor skin lesions [10].

During the COVID-19 pandemic, telemedicine has been increasingly used to bypass the severe restrictions for outpatients' visits and access to hospitals. As often happens, an urgent need (in this case the necessity to guarantee follow up for patients suffering from chronic conditions who cannot postpone their visits) became the driving force to rapidly develop and implement telemedicine and telehealth systems.
Using a widely available technology (a computer with a webcam and dedicated platforms that can ensure patients' data safety), a virtual consultation can be established between patient and clinicians in total safety.

Besides the evident convenience for $\mathrm{pa}^{-}$ tients (who can overcome the travel and parking issues and are not required to find an accompanying person), telemedicine offers some peculiar advantages, such as:

- getting a more informal assessment of mental status thanks to the opportunity to observe patients in their home environment;

- gather other social information, e.g.:

- living environment;

- interactions with family and caregivers;

- patients' lives at home [11].

The most significant question is whether telemedicine is comparable ("as good as") to in person consultation in terms of outcomes and quality. In some non-inferiority studies, tele-psychiatric outcomes were deemed as not inferior to in person care in terms of diagnosis and treatment, decreasing length of hospital stays, improving medication adherence, and reducing symptoms in conditions such as posttraumatic stress disorder on an evidenced based level [2]. Unfortunately, this comparison has not been made in other specialties.

Despite its attractivity, this tool has some obvious limitations:
${ }^{1}$ Anestesia e terapia del dolore, Ospedale di Iseo (BS) - ASST Franciacorta, Italy
Corresponding author Andrea Tinnirello andreatinny@libero.it

Received: 30 July 2021 Accepted: 30 July 2021 Published: 30 July 2021 
- elderly or low-educated patients can experience difficulties in accessing web-based applications and using technology;

- clinicians are not able to physically evaluate patients and must rely on their symptom's description;

- ethical and legal issues exist, since specific regulations on the application of telemedicine are lacking;

- reimbursement is not defined (should a virtual consultation be paid by the patient or reimbursed by national health services in the same amount as an in-person visit?).

To overcome the limitation of physical distancing, strategies on how to perform a physical evaluation with telemedicine are developing.

Recently, an American multidisciplinary expert panel, published a practical guide on how to perform an orthopedic and neurological evaluation in a virtual consultation, where the patient has to be adequately instructed on how to prepare for the consultation (adequate dressing to enable the evaluation of the anatomical region of interest, room setting, instruments and tools which should be used to perform tests) and the physician trained to ask the patient to perform specific movements and maneuvers and to observe specific signs on the screen. As a practical example, a FABER test to assess sacroiliac pathology can be performed by instructing the patient to cross leg and place ankle on opposite knee, then push the bent knee down with his hand [12].

\section{CONCLUSION}

It's the author opinion that telemedicine will find its specific role in several medical specialties. In some cases, it will be the solution to maintain constant follow up with chronic patients, thus allowing to avoid long waiting lists and increasing patients' comfort and satisfaction (as in the case of diabetes, cardiology, chronic pain management). For other specialties, it could be a sort of screening step, where, in short time, patients receive consultation and information on the need to perform specific exams before an in-person visit (e.g., orthopedic patients who receive the first consultation regarding a joint replacement).

To prevent claims and ethical issues that would limit the usage of such a helpful tool, specific guidelines regarding data protection safety, informed consent, and professional liability should be developed (using already existing regulations such as the European General Data Protection Regulation).

In an era where resources are scarce, chronic illnesses are increasing due to the aging population and the improvement of treatments (which leads to increased survival and a wider number of chronic patients requiring timely follow up) and also patients' needs and expectations are constantly increasing, telemedicine is a useful and attractive tool, which will never replace traditional patients'evaluation, but may be a substantial added value to the healthcare system.

\section{Funding}

This article has been published without the support of sponsors.

Conflicts of interests

Dr. Tinnirello has no conflict of interest to disclose regarding this work.

\section{REFERENCES}

1. Mermelstein H, Guzman E, Rabinowitz T, et al. The Application of Technology to Health: The Evolution of Telephone to Telemedicine and Telepsychiatry: A Historical Review and Look at Human Factors'.J technol behave sci 2017; 2: 5-20; https://doi.org/10.1007/s41347-017-0010-x

2. Hilty DM, Ferrer DC, Parish MB, et al. The Effectiveness of Telemental Health: A 2013 Review. Telemedicine and e-Health 2013; 19: 444-54; https://doi.org/10.1089/tmj.2013.0075

3. Lee S, Hitt WC. Clinical Applications of Telemedicine in Gynecology and Women's Health. Obstet Gynecol Clin North Am 2020; 47: 259-70; https://doi.org/10.1016/j.ogc.2020.02.002

4. Sun C, Sun L, Xi S, et al. Mobile Phone-Based Telemedicine Practice in Older Chinese Patients with Type 2 Diabetes Mellitus: Randomized Controlled Trial. JMIR Mhealth Uhealth 2019; 7: e10664; https://doi.org/10.2196/10664

5. Xu T, Pujara S, Sutton S, et al. Telemedicine in the Management of Type 1 Diabetes. Prev Chronic Dis 2018; 15: E13; https://doi.org/10.5888/pcd15.170168 
6. Hazenberg CEVB, Aan de Stegge WB, Van Baal SG, et al. Telehealth and telemedicine applications for the diabetic foot: A systematic review. Diabetes Metab Res Rev 2020; 36: e3247; https://doi.org/10.1002/dmrr.3247

7. Chen L, Cheng L, Gao W, et al. Telemedicine in Chronic Wound Management: Systematic Review And Meta-Analysis. JMIR Mhealth Uhealth. 2020; 8: e15574; https://doi. org/10.2196/15574

8. Nguyen M, Waller M, Pandya A, et al. A Review of Patient and Provider Satisfaction with Telemedicine. Curr Allergy Asthma Rep 2020; 20: 72; https://doi.org/10.1007/s11882-02000969-7

9. Polinski JM, Barker T, Gagliano N, et al. Patients' Satisfaction with and Preference for Telehealth Visits. J Gen Intern Med 2016; 31: 269-75; https://doi.org/10.1007/s11606-015-3489-x

10. Weinstein RS, Krupinski EA, Doarn CR. Clinical Examination Component of Telemedicine, Telehealth, mHealth, and Connected Health Medical Practices. Medical Clinics of North America 2018; 102: 533-44; https://doi.org/10.1016/j.mcna.2018.01.002

11. Emerick T, Alter B, Jarquin S, et al. Telemedicine for Chronic Pain in the COVID-19 Era and Beyond. Pain Med 2020; 21: 1743-8; https://doi.org/10.1093/pm/pnaa220

12. Wahezi SE, Duarte RA, Yerra S, et al.Telemedicine During COVID-19 and Beyond: A Practical Guide and Best Practices Multidisciplinary Approach for the Orthopedic and Neurologic Pain Physical Examination. Pain Physician 2020; 23: S205-S238 